\title{
Modelling immune response and drug therapy in human malaria infection
}

\author{
C. Chiyaka ${ }^{\mathrm{a} *}$, W. Garira ${ }^{\mathrm{a}}$ and S. Dube ${ }^{\mathrm{b}}$ \\ ${ }^{a}$ Department of Applied Mathematics, National University of Science and Technology, P.O. Box \\ AC 939, Ascot, Bulawayo, Zimbabwe; ${ }^{b}$ Department of Applied Biology/Biochemistry, National \\ University of Science and Technology, P.O. Box AC 939, Ascot, Bulawayo, Zimbabwe
}

(Received 14 December 2006; final version received 12 December 2007)

\begin{abstract}
A new intra-host model of malaria that describes the dynamics of the blood stages of the parasite and its interaction with red blood cells and immune effectors is proposed. Local and global stability of the disease free equilibrium are investigated. Conditions for existence and uniqueness of the endemic equilibrium are derived. An intra-host basic reproductive number is identified. We deduce that drugs based on inhibiting parasite production are more effective than those based on inhibiting merozoite invasion of erythrocytes. We extend the model to incorporate, in addition to immune response, drug therapy, following treatment with antimalarial drugs. Using stability analysis of the model, it is shown that infection can be eradicated within the host if the drug efficacy level exceeds a certain threshold value. It will persist if the efficacy is below this threshold. Numerical simulations are done to verify the analytic results and illustrate possible behaviour of the models.
\end{abstract}

Keywords: intra-host; malaria; global stability; reproductive number; efficacy

AMS Subject Classification: 34D05; 34D23; 93D20; 92B05

\section{Introduction}

Plasmodium falciparum malaria is a major cause of morbidity and mortality, largely attributable to asexual parasitaemia [33]. Transmission begins when an infected female Anopheles mosquito takes a blood meal. As the blood meal is ingested the mosquito simultaneously injects saliva containing plasmodial sporozoites into the human host. About 30 minutes later, the motile, threadlike sporozoites migrate to the liver where they infect the liver cells $[39,31,3]$. They develop into schizonts (a developmental structure that contains merozoites) which rupture and the merozoites are released, then enter the blood stream. These merozoites infect red blood cells (RBCs) and undergo asexual reproduction, which is similar to but quicker and less prolific than that in the liver cells. This occurs within the parasitophorous vacuole in the RBC [14]. After about 48 hours for $P$. falciparum the infected erythrocyte ruptures releasing daughter parasites that quickly invade fresh erythrocytes to renew the cycle $[7,22]$. This (erythrocytic) cycle maintains infection and directly generates disease symptoms [17]. Some of the merozoites that invade RBCs, instead of developing asexually, differentiate into sexual forms called gametocytes [7]. They develop through

*Corresponding author. Email: cchiyaka@nust.ac.zw 
morphologically distinct stages, designated I-V, within the host RBC [38]. Mature (stage V) gametocytes circulate in the host's bloodstream, available to feeding Anopheles mosquitoes.

Successive erythrocytic cycles result in an increase in parasitaemia and disease unless they are brought under control by the host's protective immune responses, drug therapy or until the host dies. The human immune system is a remarkably sophisticated defender of the body. It has an array of protective cells that can be mobilized to tackle an invader, with cells of the innate immune system forming the first line of defence and those of the adaptive immune system arriving later, but with extremely specific weaponry. During asexual reproduction in the RBC, the intracellular parasite, which is not free within the erythrocyte cytoplasm but resides in a parasitophorous vacuole [14], then begins to modify both the biochemical and physiological processes of the cell [24]. In the process it digests the haemoglobin which makes up around $95 \%$ of the erythrocyte cytosol. The rapid increase in the parasite biomass then activates innate immune mechanisms [14]. When the infected erythrocyte bursts, the foreign materials also activate monocytes and macrophages, releasing cytokines that stimulate other cells of the immune system and presenting foreign antigens to the rest of the immune system $[5,26]$.

Because RBCs do not express the critical molecule major histocompatibility complex class 1 (MHC-1) on their surface, the blood stage malaria parasites cannot induce the cluster of differentiation $8\left(\mathrm{CD}^{+}\right) \mathrm{T}$ cell response and, even though the response is induced by other infected tissue such as the liver, are probably not vulnerable to its cytotoxic action [40,37]. However, $\mathrm{CD}^{+}{ }^{+} \mathrm{T}$ cells are essential for immune protection against asexual blood stages in human malaria. $\mathrm{CD} 4^{+} \mathrm{T}$ cells respond to malaria antigens by proliferation and/or secretion of cytokines, e.g. interferon-gamma (INF- $\gamma$ ) or interleukin-4 (IL-4) [37]. On reinfection, the malaria primed $\mathrm{T}$ cells produce greatly increased amounts of INF- $\gamma$ which synergize with malaria glycosyphosphatidylinositol (GPI) to upregulate the production of tumour necrosis factor-alpha (TNF- $\alpha$ ). Immunity is therefore associated with the ability to regulate the production of pro-inflammatory cytokines to an intermediate level, which mediates parasite clearance while simultaneously avoiding severe pathology. Falling antigen concentration leads to a switch in the predominant $\mathrm{T}$ cell phenotype from Th1 (INF- $\gamma$ producing) to a regulatory $\mathrm{T}$ cell phenotype (IL-10 and transforming growth factor-beta (TGF- $\beta$ ) producing). These cytokines mediate anti-inflammatory response. A dynamic equilibrium is required with a pro-inflammatory effector mechanism targeting and controlling the parasite, and antiinflammatory cytokines suppressing immunopathology.

Considerable work has been done on mathematical modelling of Plasmodium falciparum infection $[33,7,22,8,40,15,16,21,30,43,18,29,19]$. Some of the intra-host models have been reviewed by Molineaux and Dietz [32]. These models do not incorporate the effect of the immune response and treatment explicitly. That is, they do not show the effect of immune effectors on merozoite invasion of erythrocytes and suppression of parasite production by antibodies. Another drawback in these models is that they do not take into account the accelerated rate of production of RBCs during a malaria infection and the loss of uninfected RBCs. From the evidence of the effects of the immune system on disease progression $[43,9,12,13,42,45,4]$, we formulate an intra-host model of malaria infection with immune response and drug therapy. The rest of the paper is organized as follows: in the following subsection we start by restating the basic intra-host model of malaria infection [16,1], which is the foundation of our model. In Section 2, we extend the basic intra-host model to incorporate the effects of immune response and further show the positivity of solutions from this new model. The intra-host basic reproductive number 
is derived in Section 3 where local and global stability analysis of the disease free equilibrium is also considered. We show the form of the endemic equilibrium and determine its stability numerically in Section 3. We further extend the model to include treatment with an antimalarial drug in Section 4. We perform the analysis of the model in Section 4 and show that the disease reproduction number of the system, when the infection is subject to both immune response and drug pressure $\left(R_{\gamma}\right)$, reduces $R_{0}$ by some factor. Implications for control of the infection, basing our arguments on the basic reproduction number, are also discussed. Numerical simulations are performed in Section 5. A brief discussion of the results concludes the paper.

\subsection{Intra-host model without immunity}

Intra-host models of malaria infection describe the dynamics of the blood stages of the parasite and their interaction with host cells which are RBCs and immune effectors [32]. One of the earliest models used to describe intra-host dynamics of malaria infection is that of Anderson et al. [1]. The mathematical model is given as

$$
\begin{gathered}
\frac{\mathrm{d} X(t)}{\mathrm{d} t}=\lambda_{X}-\beta X(t) M(t)-\mu_{X} X(t) ; \\
\frac{\mathrm{d} Y(t)}{\mathrm{d} t}=\beta X(t) M(t)-\mu_{Y} Y(t) ; \\
\frac{\mathrm{d} M(t)}{\mathrm{d} t}=r \mu_{Y} Y(t)-\mu_{\mathrm{M}} M(t)-\beta X(t) M(t) ;
\end{gathered}
$$

where $X(t)$ and $Y(t)$ are the concentrations of uninfected RBCs and infected red blood cells (IRBCs) respectively. $M(t)$ are the merozoites (parasites) that infect RBCs. $\lambda_{X}$ is the source of RBCs from the bone marrow, $\beta$ is rate of infection of RBCs, $\mu_{X}$ is the death rate of $\mathrm{RBCs}, \mu_{Y}$ is the death rate of IRBCs, $r$ is the average number of merozoites produced per each bursting IRBC and $\mu_{\mathrm{M}}$ is the death rate of merozoites.

This model does not take into account the effect of the immune system and therefore describes the worst case scenario. The intra-host basic reproductive number for a simple malaria model is defined as the number of merozoites $(r)$, released per IRBC $\times$ the initial fraction of successful merozoites [32]. This definition gives the basic reproductive number for model (1) as $R_{0}=r \beta X_{N} /\left(\mu_{\mathrm{M}}+\beta X_{N}\right)$. The reproductive number considers the fate of a single productively infected cell in an otherwise healthy individual with normal target cell levels $X_{N}=\lambda_{X} / \mu_{X}$ [6]. In a healthy individual the target cell population is regulated according to the equation $\mathrm{d} X(t) / \mathrm{d} t=\lambda_{X}-\mu_{X} X(t)$ and homeostasis is maintained at some steady state $X_{N}=\lambda_{X} / \mu_{X}>0$. The disease free equilibrium of model system (1) can easily be shown to be globally stable. We will show in Appendix A that the endemic equilibrium of system (1) is globally asymptotically stable. We note from Appendix A that the endemic equilibrium of (1) is globally stable when $\beta \lambda_{X}<\min \left(\mu_{X}, \mu_{Y}\right) \mu_{Y}$. This condition is possible for a model without immune response such as (1), where the death rate of IRBCs can be very high.

\section{Intra-host model with immune response}

We build our model from the model system (1) by including the response of the immune system. In developing the model, we also make the following assumptions:

(1) The model assumes five interacting populations at any given time $(t)$. These are RBCs $X(t)$, IRBCs $Y(t)$, merozoites $M(t)$, immune cells $B(t)$ and antibodies $A(t)$. 
(2) Due to the many different types of immune cells elicited by the presence of malaria parasites, the model assumes them to be lumped together in one population for simplicity.

(3) The RBCs are supplied from the bone marrow at a constant rate. We assume that the supply rate is accelerated by the presence of IRBCs. However, the particular mechanisms involved in the acceleration of RBCs during a malaria infection are still poorly understood [46]. They are reduced through infection by merozoites and natural death at a constant rate. The RBCs are also destroyed through phagocytosis of erythrocytes bound to merozoites.

(4) IRBCs die at a constant rate and are also killed by the presence of immune effectors. They produce free merozoites by bursting.

(5) Production rate of merozoites is reduced by immune cells. These free parasites suffer a natural death, are eliminated from circulation by immune cells and are also reduced through infecting RBCs.

(6) Immune cells are assumed to have a per capita rate of production and their production is stimulated by the presence of IRBCs and merozoites. They are reduced (deactivated) at a constant rate.

(7) Antibodies that block invasion of RBCs proliferate in the presence of merozoites. Proliferation of antibodies is dependent only on merozoites since we assume only antibodies that block infection. Antibodies decay at a constant rate.

(8) All parameters are positive.

These assumptions lead to the following system of differential equations which describe the interaction between uninfected RBCs, IRBCs, merozoites, immune cells (which include $\mathrm{CD}^{+}{ }^{+} \mathrm{T}$ cells, dendritic cells, macrophages, etc.), and antibodies:

$$
\begin{gathered}
\frac{\mathrm{d} X(t)}{\mathrm{d} t}=\lambda_{X}+\sigma Y(t)-\frac{\beta X(t) M(t)}{1+c_{0} A(t)}-\mu_{X} X(t)-\omega X(t) M(t) B(t) ; \\
\frac{\mathrm{d} Y(t)}{\mathrm{d} t}=\frac{\beta X(t) M(t)}{1+c_{0} A(t)}-\mu_{Y} Y(t)-\kappa_{Y} B(t) Y(t) ; \\
\frac{\mathrm{d} M(t)}{\mathrm{d} t}=\frac{r \mu_{Y} Y(t)}{1+c_{1} B(t)}-\mu_{\mathrm{M}} M(t)-\kappa_{\mathrm{M}} B(t) M(t)-\frac{\beta X(t) M(t)}{1+c_{0} A(t)} ; \\
\frac{\mathrm{d} B(t)}{\mathrm{d} t}=\lambda_{B}+B(t)\left(\rho_{Y} \frac{Y(t)}{k_{0}+Y(t)}+\rho_{\mathrm{M}} \frac{M(t)}{k_{1}+M(t)}\right)-\mu_{B} B(t) ; \\
\frac{\mathrm{d} A(t)}{\mathrm{d} t}=\eta B(t) \frac{M(t)}{k_{1}+M(t)}-\mu_{A} A(t) .
\end{gathered}
$$

The dynamics of RBCs, $X(t)$ is described by Equation (2.1). The first term on the right side of Equation (2.1) represents the rate of supply of RBCs from the bone marrow [31]. The second term represents the recruitment of RBCs at a rate $\sigma$ proportional to IRBCs where $0 \leq \sigma<1$. RBC production is accelerated during a malaria infection, but the particular mechanisms involved are still poorly understood [46]. One analysis reported 
an average of $37 \%$ increase in RBC production in adult first-time $P$. falciparum patients [23]. The third term represents infection of RBCs by merozoites. $\beta X(t) M(t)$ models the rate at which free merozoites infect RBCs where $\beta$ is rate of infection. Antibodies specific to malaria parasites (merozoites) inhibit invasion of erythrocytes by merozoites [42,2]. The term $f\left(A(t), c_{0}\right)=1 /\left(1+c_{0} A(t)\right)$ represents the role of antibodies in controlling parasitaemia, where $c_{0}$ is the efficiency of antibodies in reducing erythrocytic invasion. Antibodies bind to the epitopes on the Plasmodium parasite, thereby disabling the parasite, making its entry into the RBC difficult. As the antibody level increases $(A(t) \rightarrow \infty)$, $f\left(A(t), c_{0}\right) \rightarrow 0$. This means that an increase in the number of antibodies reduces the rate of infection of RBCs by merozoites. Conversely, low numbers of antibodies increase the rate of infection of RBCs by merozoites. That is as $A(t) \rightarrow 0, f\left(A(t), c_{0}\right) \rightarrow 1$. The fourth term in Equation (2.1) is the natural death rate of RBCs, since their average life span is $1 / \mu_{X}$. The last term describes the destruction of RBCs through phagocytosis of erythrocytes bound to merozoites [23]. Equation (2.2) models the dynamics of infected (parasitized) RBCs, $Y(t)$. The first term on the right hand side of Equation (2.2) is a gain term for IRBCs from the loss term in Equation (2.1). The second term is the rate at which IRBCs are lost through death. The last term represents the rate at which IRBCs are killed directly by immune cells [12,42]. The parameter $\kappa_{Y}$ represents immunosensitivity of IRBCs [32].

The rate of change of merozoites is described by Equation (2.3). The first term on the right hand side of Equation (2.3) is the source of free merozoites which are released when IRBCs burst $[7,22]$. Their source is the second term in Equation (2.2). IRBCs burst during death, hence the number of merozoites produced depends on the death rate of IRBCs. An average of $r$ merozoites are released per each bursting IRBC. Rate of parasite production (multiplication) by infected cells is suppressed by immune cells with a factor $f\left(B(t), c_{1}\right)=1 /\left(1+c_{1} B(t)\right)$. Immune cells such as $\gamma \delta^{+}$T cells expand during the early stages of infection by $P$. falciparum [10]. The expanded or activated T-cell populations express mRNA for TNF and IFN- $\gamma$ [43] and can inhibit parasite growth [43,10]. $c_{1}$ is the efficiency of immune cells in suppressing parasite production. So as $B(t) \rightarrow \infty, f\left(B(t), c_{1}\right) \rightarrow 0$ meaning as immune cells increase, rate of parasite production is reduced. Conversely, as immune cells decrease, parasite production by IRBCs increases, thus as $B(t) \rightarrow 0, f\left(B(t), c_{1}\right) \rightarrow 1$. We note however that for malaria, a dynamic equilibrium is required between pro-inflammatory and anti-inflammatory cytokines to suppress immunopathology. The second term is the loss of merozoites through natural death and the third term is the rate at which merozoites are removed by immune cells. IFN- $\gamma$ and $\mathrm{CD} 4^{+} \mathrm{T}$ cells activate macrophages to phagocytose intra-erythrocytic parasites and free merozoites [42]. $\kappa_{\mathrm{M}}$ is immunosensitivity of merozoites. The last term is the loss of merozoites through infection of RBCs.

Equation (2.4) models the population of the immune cells (e.g. macrophages, natural killer cells, $\mathrm{CD}^{+}{ }^{\mathrm{T}}$ cells). The first term on the right hand side of Equation (2.4) is the source term for immune cells (combined term for different immune cells). This is followed by the stimulation term for immune cells in the presence of IRBCs and merozoites. $\rho_{Y}$ and $\rho_{\mathrm{M}}$ represent the immunogenicity of IRBCs and merozoites respectively [32]. $k_{0}$ is the density of IRBCs at which immune cells grow at rate $\rho_{Y} / 2$, that is half their maximum rate [13] in the absence of merozoites and $k_{1}$ is the density of merozoites [25] at which immune cells grow at rate $\rho_{\mathrm{M}} / 2$ in the absence of IRBCs. $\mu_{B}$ is the natural death rate of the immune cells. The population of antibodies is described by Equation (2.5). The first term on the right hand side of Equation (2.5) is the stimulation term for antibodies. Antibodies that inhibit invasion of RBCs by merozoites are secreted by immune cells in the presence of merozoites. These antibodies against the merozoite surface proteins (MSPs) are a major component of the invasion inhibitory response in humans [36]. $\eta$ is the maximum rate 
of increase of antibodies, $k_{1}$ is the density of merozoites at which antibodies reach half their maximum value $(\eta / 2)$ and $\mu_{A}$ is the rate at which the antibodies decay.

Unlike Equation (2.4) where we include a natural source term, $\lambda_{B}$ for immune cells, we do not include a source term for antibodies because we assume that the only source of antibodies against merozoites in a non-immune human (a human who has no previous exposure to malaria infection) are immune cells.

The solutions of the model (2) are shown to be always positive, which means that the model is biologically feasible. Positivity of solutions is shown in Appendix B. It can easily be shown that all solutions of system (2) initiating in $I R_{+}^{5}$ are bounded and eventually enter the attracting set

$$
\Phi=\left\{(X, Y, M, B, A) \in \mathbb{R}_{+}^{5}: X, Y, M, B, A<Q\right\} .
$$

\section{Analysis of the model}

In model system (2), some parameters have biological meaning and can be well estimated and others are mathematical constructs imposed by our understanding of the immune system. To examine such a model for its qualitative behaviour and estimate predicted outcome we examine the following:

(a) the system's equilibria and/or conditions for equilibria,

(b) the stability of the equilibrium points,

(c) the effect of treatment.

\subsection{Intra-host basic reproductive number and stability analysis}

The intra-host basic reproductive number $R_{0}$ of the malaria parasite is defined as the number of secondary IRBCs produced per primary IRBC in a host at the onset of infection [32]. If $R_{0}<1$, then on average an IRBC produces less than one new IRBC and the infection cannot grow. If however $R_{0}>1$, then on average each IRBC produces more than one new IRBC and infection is maintained. Following the method of the next generator approach [44], the matrices $F$ and $V^{-1}$ are given as:

$$
\begin{gathered}
F=\left(\begin{array}{cc}
0 & \beta \frac{\lambda_{X}}{\mu_{X}} \\
0 & 0
\end{array}\right), \\
V^{-1}=\left(\begin{array}{cc}
\frac{1}{\mu_{Y}+\kappa_{Y}\left(\lambda_{B} / \mu_{B}\right)} \\
\frac{r \mu_{Y}}{\left(1+c_{1}\left(\lambda_{B} / \mu_{B}\right)\right)\left(\mu_{Y}+\kappa_{Y}\left(\lambda_{B} / \mu_{B}\right)\right)\left(\mu_{\mathrm{M}}+\kappa_{\mathrm{M}}\left(\lambda_{B} / \mu_{B}\right)+\beta\left(\lambda_{X} / \mu_{X}\right)\right)} & \frac{1}{\left(\mu_{\mathrm{M}}+\kappa_{\mathrm{M}}\left(\lambda_{B} / \mu_{B}\right)+\beta\left(\lambda_{X} / \mu_{X}\right)\right)}
\end{array}\right) .
\end{gathered}
$$

The product $F V^{-1}$ is called the next generation matrix [8]. The reproductive number $R_{0}$, is the dominant eigenvalue of $F V^{-1}$. For the model system (2), we get

$$
R_{0}=\frac{r \mu_{Y} \beta \lambda_{X} \mu_{B}^{3}}{\left(\mu_{B}+c_{1} \lambda_{B}\right)\left(\mu_{B} \mu_{Y}+\kappa_{Y} \lambda_{B}\right)\left(\mu_{B} \mu_{\mathrm{M}} \mu_{X}+\mu_{X} \kappa_{\mathrm{M}} \lambda_{B}+\mu_{B} \beta \lambda_{X}\right)} .
$$


The expression for $R_{0}$ in (3.1) can be written as

$$
\frac{\beta \bar{X}}{1+c_{0} \bar{A}} \times \frac{r \mu_{Y}}{1+c_{1} \bar{B}} \times\left(\mu_{\mathrm{M}}+\kappa_{\mathrm{M}} \bar{B}+\frac{\beta \bar{X}}{1+c_{0} \bar{A}}\right)^{-1} \times\left(\mu_{Y}+\kappa_{Y} \bar{B}\right)^{-1},
$$

where the first term in (3.2) is the rate at which a merozoite introduced into a completely susceptible RBC population (where $X=\bar{X}, B=\bar{B}, A=\bar{A}$ ) infects a RBC. The second term is the number of merozoites produced by the IRBC when it bursts. The third term is the life span of each produced merozoite and the last term is the life span of the IRBC.

The model system given by Equation (2) has two steady states, the disease-free state denoted by

$$
E_{0}=(\bar{X}, \bar{Y}, \bar{M}, \bar{B}, \bar{A})=\left(\frac{\lambda_{X}}{\mu_{X}}, 0,0, \frac{\lambda_{B}}{\mu_{B}}, 0\right)
$$

and the endemic state denoted by

$$
E_{\mathrm{e}}=\left(X^{*}, Y^{*}, M^{*}, B^{*}, A^{*}\right) \text {. }
$$

To bring the infection (parasitaemia) under control we seek conditions on the parameters of the transmission process that will guarantee the existence of a stable diseasefree state. These conditions are summarized in Appendix C and Appendix D.

\subsection{The endemic equilibrium and its stability}

When $R_{0}>1$, the condition for the stability of the disease-free equilibrium is violated and besides the disease-free equilibrium, the system of Equation (2) has an endemic equilibrium point, $E_{\mathrm{e}}=\left(X^{*}, Y^{*}, M^{*}, B^{*}, A^{*}\right)$. The explicit form of the endemic equilibrium is quite cumbersome to be produced here, therefore we shall only show its existence numerically for a certain range of parameter values and perform its stability analysis. Numerical simulations in Section 5 also confirm the existence of an endemic equilibrium for the parameters used. We show numerically that when $R_{0}<1$, the disease free state is stable and when $R_{0}>1$, the system attains an endemic equilibrium which is stable and the disease-free state becomes unstable. The bifurcation diagram from numerical simulations to illustrate this is shown in Figure 1 and it was produced by using the fourth order Runge Kutta method coded in the $C^{++}$programming language. It is obtained by varying $r$, the average number of merozoites produced per each bursting IRBC, while the other parameters are fixed. This shows that there exists a critical value,

$$
r^{c}=\frac{\left(\mu_{B}+c_{1} \lambda_{B}\right)\left(\mu_{B} \mu_{Y}+\kappa_{Y} \lambda_{B}\right)\left(\mu_{B} \mu_{\mathrm{M}} \mu_{X}+\mu_{X} \kappa_{\mathrm{M}} \lambda_{B}+\mu_{B} \beta \lambda_{X}\right)}{\beta \lambda_{X} \mu_{Y} \mu_{B}^{3}}
$$

above which the infection will persist. This is obtained when $R_{0}=1$ in Equation (3.1). Therefore the uninfected steady state is stable if $r<r^{c}$.

\section{Intra-host model with immune response and drug therapy}

We extend the model to incorporate effects of the drug. Antimalarial drugs taken prophylactically or during infection (blood schizonticides) concentrate particularly in parasitized erythrocytes. The drug diffuses into parasite lysosomal compartments and becomes protonated in the acidic environment within, so it cannot pass out through the 


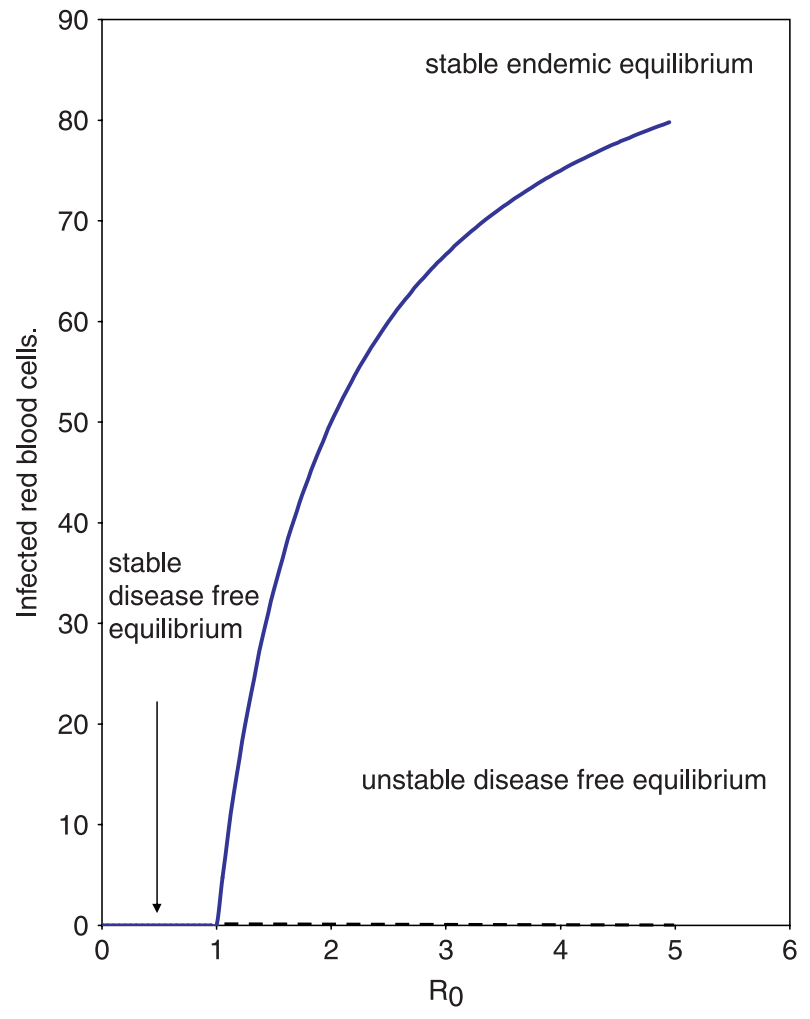

Figure 1. Bifurcation diagram showing the variation in $Y$ with $R_{0}$ as bifurcation parameter. Stability is shown by bold lines and dashed lines indicate unstable equilibrium. The disease free equilibrium is stable when $R_{0}<1$ and unstable otherwise. The endemic equilibrium exists and is stable when $R_{0}>1$. The bifurcation diagram was obtained by varying $r$, the average number of merozoites produced per IRBC while keeping all the other parameters fixed at numerical values given as $\lambda_{X}=41664, \sigma=0.009, \beta=7, c_{0}=0.6, \mu_{X}=0.008, \mu_{Y}=1.0, \kappa_{Y}=0.9, \kappa_{\mathrm{M}}=0.3$, $c_{1}=0.85, \mu_{\mathrm{M}}=3.0, \lambda_{B}=30, \rho_{Y}=0.05, \rho_{\mathrm{M}}=0.07, k_{0}=2000, k_{1}=1500, \mu_{B}=0.08, \mu_{A}=0.06$, $\eta=0.8$ and $\omega=0.0025$.

membrane. It raises the $\mathrm{pH}$ of lysosome, inhibiting the polymerase that converts toxic free haem to a harmless by-product. It prevents digestion of haemoglobin by parasites, reducing its supply of amino acids and therefore makes the parasite survival and development difficult. If the drug is administered, then the burst size $r$ becomes $(1-\gamma) r$ where $\gamma$ is drug efficacy and is assumed to lie between zero, meaning totally ineffective, and one, meaning $100 \%$ effectiveness. The drug efficacy in this context is the probability with which chloroquine inhibits parasite growth inside IRBCs. The model system then becomes

$$
\begin{gathered}
\frac{\mathrm{d} X(t)}{\mathrm{d} t}=\lambda_{X}+\sigma Y(t)-\frac{\beta X(t) M(t)}{1+c_{0} A(t)}-\mu_{X} X(t)-\omega X(t) M(t) B(t) ; \\
\frac{\mathrm{d} Y(t)}{\mathrm{d} t}=\frac{\beta X(t) M(t)}{1+c_{0} A(t)}-\mu_{Y} Y(t)-\kappa_{Y} B(t) Y(t) ;
\end{gathered}
$$




$$
\begin{gathered}
\frac{\mathrm{d} M(t)}{\mathrm{d} t}=\frac{(1-\gamma) r \mu_{Y} Y(t)}{1+c_{1} B(t)}-\mu_{\mathrm{M}} M(t)-\kappa_{\mathrm{M}} B(t) M(t)-\frac{\beta X(t) M(t)}{1+c_{0} A(t)} \\
\frac{\mathrm{d} B(t)}{\mathrm{d} t}=\lambda_{B}+B(t)\left(\rho_{Y} \frac{Y(t)}{k_{0}+Y(t)}+\rho_{\mathrm{M}} \frac{M(t)}{k_{1}+M(t)}\right)-\mu_{B} B(t) \\
\frac{\mathrm{d} A(t)}{\mathrm{d} t}=\eta B(t) \frac{M(t)}{k_{1}+M(t)}-\mu_{A} A(t) .
\end{gathered}
$$

The Equations (4.1), (4.2), (4.4) and (4.5) are the same as (2.1), (2.2), (2.4) and (2.5) respectively. Equation (4.3) is a modification of Equation (2.3) to incorporate the effects of antimalarial drugs.

The model system (4) has two equilibrium states, the disease free state

$$
E^{0}=(\underline{X}, \underline{Y}, \underline{M}, \underline{B}, \underline{A})=\left(\frac{\lambda_{X}}{\mu_{X}}, 0,0, \frac{\lambda_{B}}{\mu_{B}}, 0\right),
$$

and the endemic equilibrium $E^{\mathrm{e}}=(\tilde{X}, \tilde{Y}, \tilde{M}, \tilde{B}, \tilde{A})$, and due to the complexity of our model, the endemic equilibrium is shown numerically so that it exists for the parameter values used.

For the disease-free equilibrium to be locally stable all the eigenvalues of the Jacobean matrix evaluated at the disease-free state should be negative. The eigenvalues correspond to the roots of the characteristic Equation $|\mathbf{J}-z \mathbf{I}|=0$, where $\mathbf{J}$ is the Jacobian matrix, $z$ is the eigenvalue and $\mathbf{I}$ is a $5 \times 5$ identity matrix. The characteristic equation is:

$$
\begin{gathered}
\left(z^{2}+\left(\mu_{\mathrm{M}}+\mu_{Y}+\kappa_{Y} \frac{\lambda_{B}}{\mu_{B}}+\kappa_{\mathrm{M}} \frac{\lambda_{B}}{\mu_{B}}+\beta \frac{\lambda_{X}}{\mu_{X}}\right) z+\left(\mu_{\mathrm{M}}+\kappa_{\mathrm{M}} \frac{\lambda_{B}}{\mu_{B}}+\beta \frac{\lambda_{X}}{\mu_{X}}\right)\left(\mu_{Y}+\kappa_{Y} \frac{\lambda_{B}}{\mu_{B}}\right)\right. \\
\left.-\frac{(1-\gamma) r \mu_{Y} \beta \lambda_{X}}{\mu_{X}\left(1+c_{1}\left(\lambda_{B} / \mu_{B}\right)\right)}\right) \times\left(-\mu_{X}-z\right)\left(-\mu_{B}-z\right)\left(-\mu_{A}-z\right)=0 .
\end{gathered}
$$

Clearly three of the roots, $-\mu_{X},-\mu_{B}$ and $-\mu_{A}$, are negative. We use Routh-Hurwitz condition to establish that all roots are negative if the condition

$$
\left(\mu_{\mathrm{M}}+\kappa_{\mathrm{M}} \frac{\lambda_{B}}{\mu_{B}}+\beta \frac{\lambda_{X}}{\mu_{X}}\right)\left(\mu_{Y}+\kappa_{Y} \frac{\lambda_{B}}{\mu_{B}}\right)-\frac{(1-\gamma) r \mu_{Y} \beta \lambda_{X}}{\mu_{X}\left(1+c_{1} \frac{\lambda_{B}}{\mu_{B}}\right)}>0,
$$

is satisfied. We then deduce the reproductive number $R_{\gamma}$ when antimalarial drugs are administered to be

$$
R_{\gamma}=\frac{(1-\gamma) r \mu_{Y} \beta \lambda_{X} \mu_{B}^{3}}{\left(\mu_{B}+c_{1} \lambda_{B}\right)\left(\mu_{B} \mu_{Y}+\kappa_{Y} \lambda_{B}\right)\left(\mu_{B} \mu_{\mathrm{M}} \mu_{X}+\mu_{X} \kappa_{\mathrm{M}} \lambda_{B}+\mu_{B} \beta \lambda_{X}\right)}=R_{0}(1-\gamma)
$$

Furthermore, it can be seen that at least one of these eigenvalues has a positive real part if $R_{\gamma}>1$. Thus we have established the following result:

LEMMA. The disease free equilibrium $E^{0}$ of (4) is locally asymptotically stable if $R_{\gamma}<1$ and unstable if $R_{\gamma}>1$. 


\subsection{Implications for control}

Knowledge of factors that limit parasite numbers offers hope of better-designed treatment and intervention strategies [15,30,32], as well as providing information on selective forces that have moulded parasite life-history strategies [20]. The intra-host basic reproductive number $R_{0}$ is a key parameter of asexual parasitaemia, crucial to calculations concerning its control by any mechanism, natural or artificial. From the formula of $R_{0}$ in (3.1), we deduce the following: (a) reduction in the transmission rate $\beta$ reduces the reproductive number. (b) Increasing the rate at which immune cells suppress parasite production is more effective in the control of the disease than increasing the rate at which antibodies inhibit invasion of parasites into erythrocytes, since the parameter $c_{1}$, efficiency of immune cells appears in $R_{0}$ and $c_{0}$, efficiency of antibodies does not. (c) Since $\mu_{B}$ is a cubed term in the expression for $R_{0}$, increasing $\mu_{B}$ greatly increases $R_{0}$.

Biologically speaking, $R_{\gamma}$ measures the number of secondary infections generated by a single parasitized RBC in an environment where antimalarial drugs are used as a control strategy. The primary focus of drug therapy is on the possibility of clearing the parasites. If $R_{\gamma}<1$, then parasites are cleared. Using the critical point $R_{\gamma}=1$, we find the critical drug efficacy $\gamma^{c}$ that is required for parasite clearance to be

$$
\gamma^{c}=1-\frac{1}{R_{0}} .
$$

$R_{0}$ is the reproductive number when there is immune response only. If immune response fails to clear the parasites $\left(R_{0}>1\right)$, the drug is then administered, with the aim of reducing $R_{0} . R_{\gamma}$ is always less than $R_{0}$ for all values of $\gamma$ in the range $0<\gamma<1 . R_{\gamma}=R_{0}$ only when $\gamma=0$. This implies that the drug is completely ineffective. $R_{\gamma}<1$ whenever $\gamma>\gamma^{c}$, thus malaria parasites can be eradicated from an infected individual if $\gamma>\gamma^{c}$.

\section{Numerical simulation}

To observe the dynamics of the model over time we integrated the system of equations, using the fourth order Runge-Kutta methods in the $C^{++}$programming language. For computer runs we set the initial densities of RBCs, IRBCs, merozoites, immune cells and antibodies at 500, 5, 50, 30, and 10 respectively. Time is in days. The fixed parameters used are shown in Table 1 . The value of the reproductive number $R_{0}=22.8$.

Choosing parameter values characteristics of the in vivo situation is difficult. Many of the parameters in our model, as shown in Table 1, are estimated. Even those that have been quoted may not be as accurate as we need for quantitative predictions. However, our main thrust is on the overall effect they have on the basic reproductive number, which is the basis for an infection to persist or be eradicated.

The graphs in Figure 2 show the behaviour of model system (2). It shows that the populations of uninfected RBCs, IRBCs and merozoites reach a steady endemic state since $R_{0}>1$. Figure 3 shows the behaviour of model system (4) where $\gamma=0.97$. The graphs show that the infection can be eradicated if the efficacy of the drug $\gamma>\gamma^{c}$. If the immune system alone fails to clear the infection then a drug of suitable efficacy can accomplish the task of bringing the infection to disease-free levels. Figure 4 shows that as the death rate of immune cells, $\mu_{B}$, increases there is an increase in the number of IRBCs and merozoites, while the uninfected RBCs decrease.

Figure 5 shows the behaviour of (a) RBCs (b) uninfected RBCs and (c) merozoites of model system (4.1)-(4.5) as the rate at which RBCs are eliminated due to the effect 
Table 1. Table showing numerical values of parameters used in the simulations performed where c are cells, $\mathrm{d}$ are days and $\mathrm{m}$ are merozoites.

\begin{tabular}{|c|c|c|c|c|}
\hline Parameter & Symbol & Value & Units & Source \\
\hline Supply rate of RBC & $\lambda_{X}$ & 41664 & c $\mu l^{-1} d^{-1}$ & {$[1]$} \\
\hline Rate of recruitment of $\mathrm{RBC}$ & $\sigma$ & 0.009 & $c \mathrm{~d}^{-1}$ & Estimated \\
\hline Rate of infection & $\beta$ & 0.08 & $\mathrm{M} \mu \mathrm{l}^{-1} \mathrm{~d}^{-1}$ & Estimated \\
\hline Efficiency of antibodies & $c_{O}$ & 0.6 & $\mu l$ & Estimated \\
\hline Death rate of $\mathrm{RBCs}$ & $\mu_{X}$ & 0.8 & $d^{-1}$ & Estimated \\
\hline Death rate of IRBCs & $\mu_{Y}$ & 1.0 & $\mathrm{~d}^{-1}$ & Estimated \\
\hline Immunosensitivity of IRBC & $\kappa_{Y}$ & 0.9 & $\mathrm{c} \mu \mathrm{ld}^{-1}$ & Estimated \\
\hline Rate at which RBCs are eliminated & $\omega$ & $1.2 \times 10^{-5}$ & $c d^{-1}$ & Estimated \\
\hline $\begin{array}{l}\text { Merozoites released per each bursting } \\
\text { IRBC }\end{array}$ & $r$ & 16 & $\mathrm{~m} \mu \mathrm{l}^{-1}$ & {$[5,21,9,46]$} \\
\hline $\begin{array}{l}\text { Rate at which parasite production } \\
\text { is suppressed }\end{array}$ & $c_{1}$ & 0.85 & c $\mu l$ & Estimated \\
\hline Death rate of merozoites & $\mu_{\mathrm{M}}$ & 3.0 & $\mathrm{~d}^{-1}$ & Estimated \\
\hline Supply rate of immune cells & $\lambda_{B}$ & 30 & $\mathrm{c} \mu \mathrm{d} \mathrm{d}^{-1}$ & Estimated \\
\hline Immunogenicity of IRBCs & $\rho_{Y}$ & 0.05 & $\mathrm{~d}^{-1}$ & Estimated \\
\hline Immunogenecity of merozoites & $\rho_{\mathrm{M}}$ & 0.05 & $d^{-1}$ & Estimated \\
\hline $\begin{array}{l}\text { Stimulation constant for immune cells } \\
\text { due to IRBCs }\end{array}$ & $k_{0}$ & 2000 & $\mathrm{c} \mu \mathrm{l}^{-1}$ & Estimated \\
\hline $\begin{array}{l}\text { Stimulation constant for immune cells } \\
\text { due to merozoites }\end{array}$ & $k_{1}$ & 1500 & $\mathrm{~m} \mu \mathrm{l}^{-1}$ & Estimated \\
\hline Death rate of immune cells & $\mu_{B}$ & 1.53 & $\mathrm{~d}^{-1}$ & Estimated \\
\hline Immunosensitivity of merozoites & $\kappa_{\mathrm{M}}$ & 0.3 & $\mathrm{~m} \mu \mathrm{ld}^{-1}$ & Estimated \\
\hline Deterioration rate of antibodies & $\mu_{A}$ & 0.4 & $\mu 1^{-1}$ & Estimated \\
\hline Maximum rate of increase of antibodies & $\eta$ & 0.6 & $\mu 1^{-1}$ & Estimated \\
\hline
\end{tabular}

of involvement $\omega$ is varied from 0.0 to 0.08 in steps of 0.02 . The arrows show the direction of increase of $\omega$. The graphs show that as $\omega$ increases, there is a corresponding decrease in the populations of RBCs, IRBCs and antibodies. A decrease in RBC population gives a corresponding decrease in the number of IRBCs which makes the number of immune cells and antibodies stimulated decrease as well.

\section{Discussion}

A model of the immune response to Plasmodium falciparum infection was developed and extended to include treatment with antimalarial drugs. As a first step towards more realistic modelling of immune regulation, we introduce the terms for the effects of immune effectors on merozoite invasion of RBCs and parasite production within a RBC. In addition, we allow the RBCs to be eliminated through the effect of involvement [35]. Our mathematical analysis yielded a generalization of the intra-host basic reproductive number from which the most effective control strategies that should aid in assessing interventions (drugs and vaccines) are deduced. Our results show that without any treatment the most effective part of the immune response in its mission to clear parasites is its ability to inhibit parasite growth in erythrocytes. It is also more effective to increase the death rate of IRBCs than the death rate of merozoites by immune cells. This is because one IRBC is capable of producing about 16 merozoites, so killing it will prevent the survival of 16 merozoites. Most of the available malaria drugs act by retarding development of parasites. Drugs that kill IRBCs directly, together with those that retard the development of parasites, should also be made available to make the treatment more effective. For the extended model, with treatment, we have 

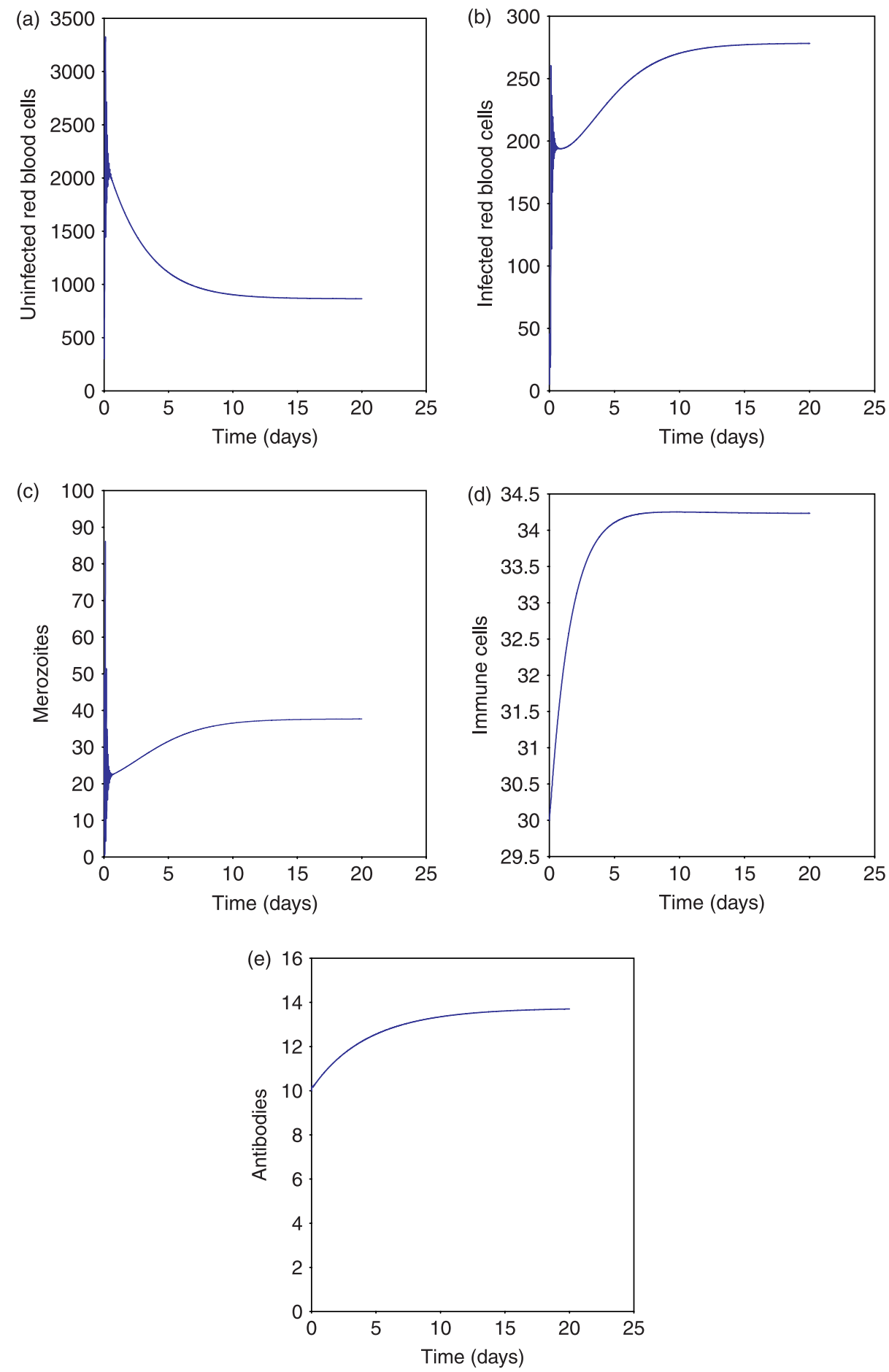

Figure 2. Graphs that show the behaviour of the model with immune response only. The dynamics of (a) uninfected RBCs, (b) IRBCs, (c) merozoites, (d) immune cells and (e) antibodies. 

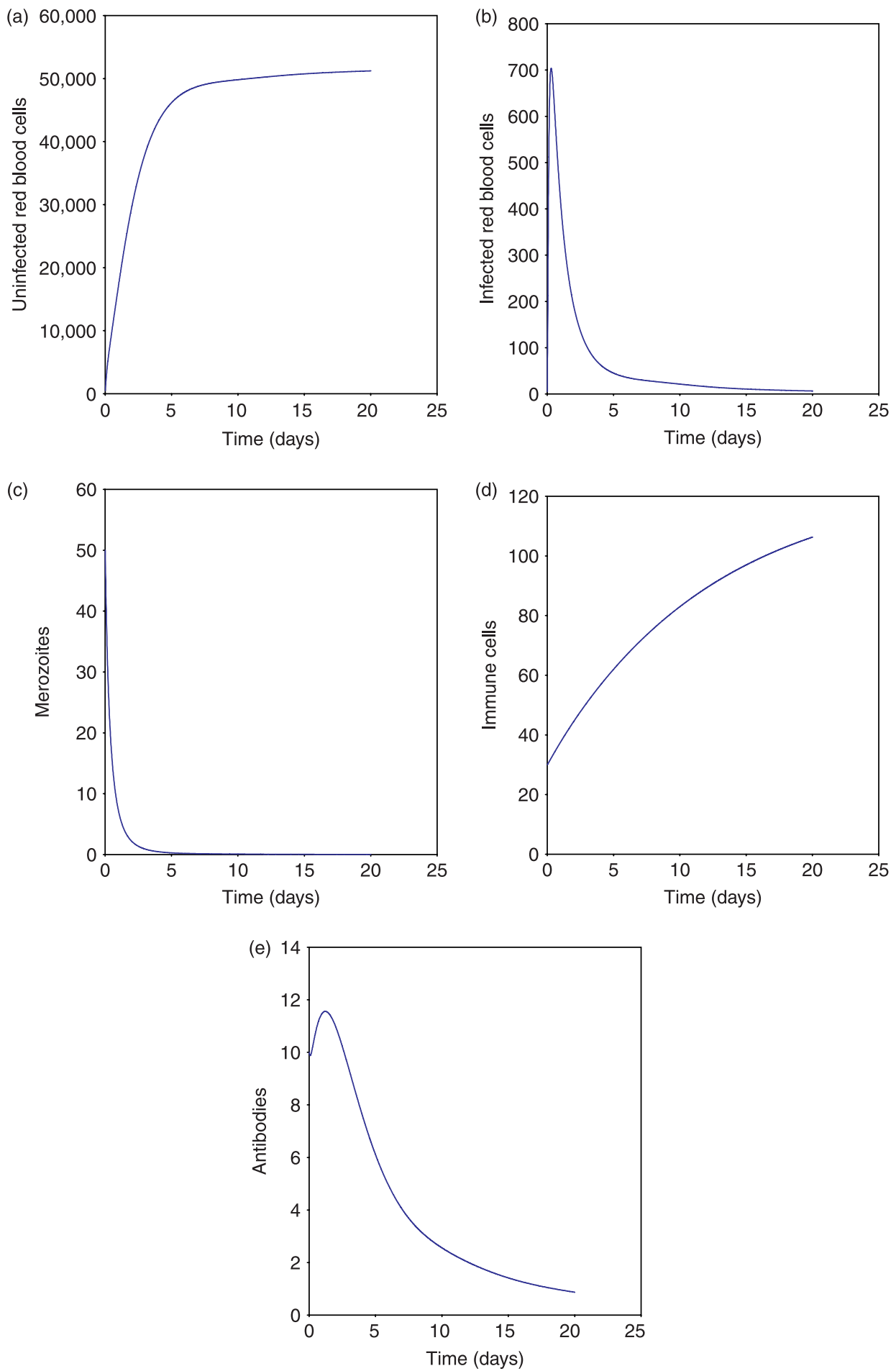

Figure 3. Graphs that show the behaviour of the model with immune response and drug therapy. The dynamics of (a) uninfected RBCs, (b) IRBCs, (c) merozoites, (d) immune cells and (e) antibodies. 

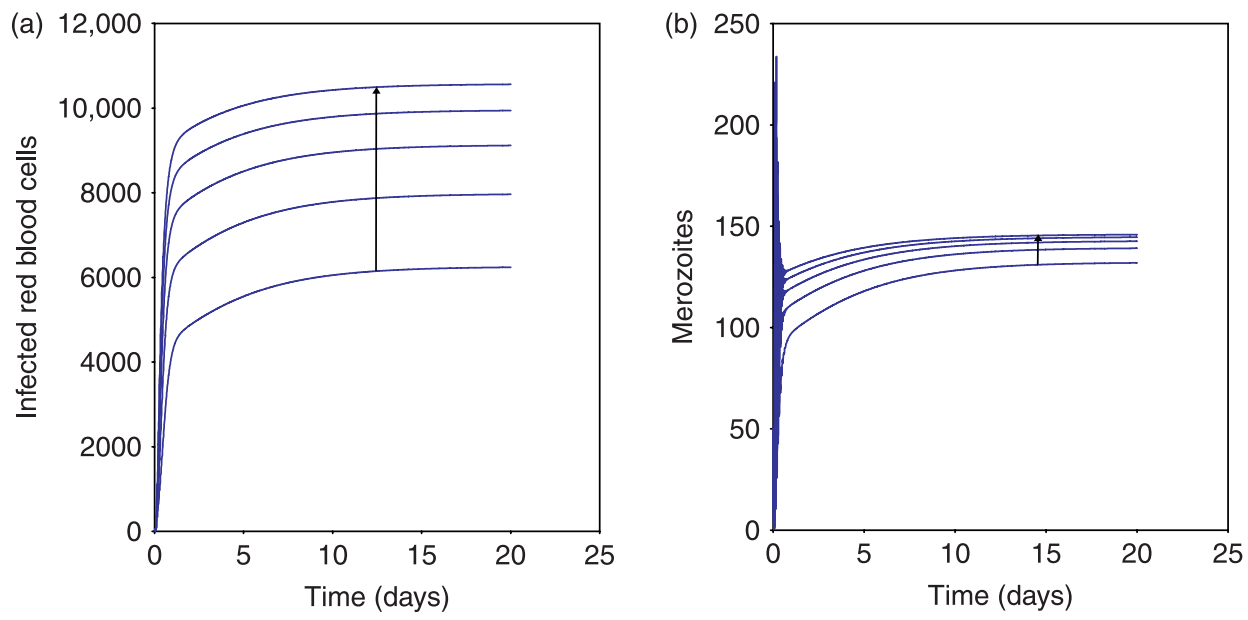

Figure 4. Graphs that show the behaviour of (a) uninfected RBCs and (b) merozoites. They were obtained by varying the death rate of immune cells $\mu_{B}$ from 0.5 to 2.5 in steps of 0.5 , while keeping the other parameters constant. The arrows show the direction of increase of $\mu_{B}$.

shown that parasites can only be cleared from an infected individual if $\gamma>\gamma^{c}$. This implies that for a very large reproductive number, a drug with an efficacy of $\gamma \approx 1$ is required to treat the infection. This might be one of the reasons why for a person from a non endemic area the infection is more virulent and, if treatment is delayed, then death might be inevitable.

We have also observed that if there are mechanisms in the body that increase the death rate of immune cells then there is an increase in the severity of the infection. This shows that for a person infected by pathogens that kill some of the immune cells, like the human immunodeficiency virus which kills the $\mathrm{CD}^{+}{ }^{+} \mathrm{T}$ cells [41], or Mycobacterium tuberculosis which kills the macrophages, then that person is infected by P. falciparum parasite, the immune system is compromised and the person suffers severe malaria if not treated in time. People who suffer from diseases such as acquired immunodeficiency syndrome or tuberculosis should not delay in seeking medical attention when symptoms of malaria start to appear. Analysis also yielded a well-known result that the effect of involvement decreases the number of RBCs, which is believed to be one of the causes of anaemia in malaria.

Our mathematical analysis is useful in explaining some of the observed patterns during a malaria infection. This gives our model the ability to provide a basic representation of the complex web that accompanies an intruding malaria parasite during the erythrocytic stages. For people moving to endemic areas, prophylactic drugs should be taken a week before departure so that an adequate concentration is attained in the blood before exposure to parasites and continued a month after return to kill any parasites incubating in the liver as they emerge.

Drug resistance has been a major cause of resurgence of the disease and will be considered elsewhere. The complexity of the parasite, the different forms it assumes in the body and the possibility of the patient being infected by several strains concurrently make model predictions more difficult. Sporozoites are a target for vaccine development but are able to replace their coats, varying the antigens they present. Relevant data should be made available to be used in model validation. Thus one role of modelling is to point out where further quantitative measurements can improve our understanding of the malaria disease process. 

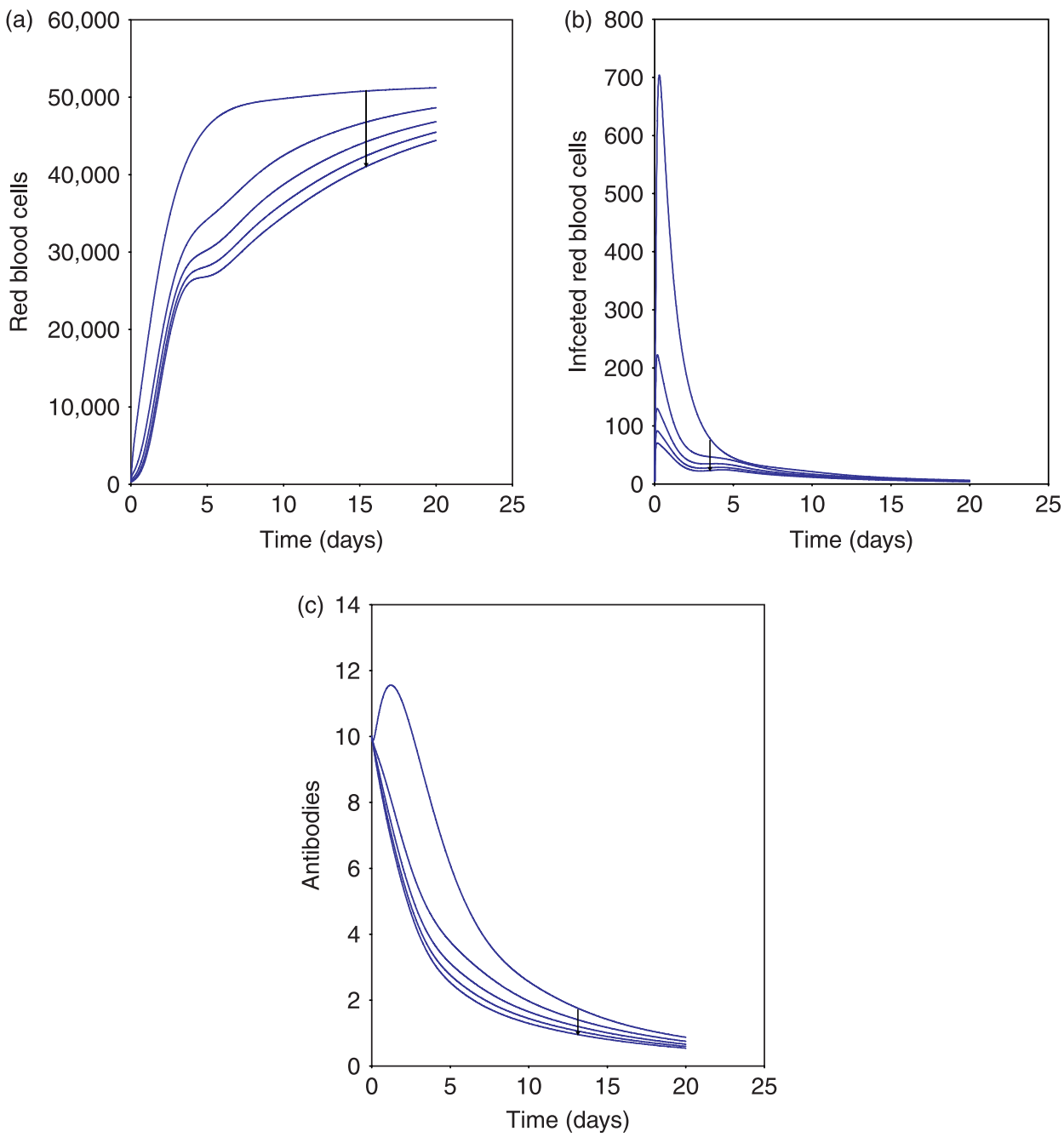

Figure 5. Graphs that show the behaviour of (a) uninfected RBCs, (b) IRBCs and (c) antibodies. The graphs are obtained by varying the rate at which uninfected RBCs are eliminated, $\omega$ from 0.0 to 0.08 in steps of 0.02 , while keeping the other parameters constant. The arrows show the direction of increase of $\omega$.

\section{Acknowledgements}

The authors would like to thank the two anonymous referees for their constructive comments which have greatly improved the paper. The authors also acknowledge financial support from Eagle Insurance Company, Zimbabwe. C. Chiyaka would like to acknowledge financial support by National University of Science and Technology through a Staff Development Scholarship. We also want to thank Professors Hagai Ginsburg and Klaus Dietz for sending us literature on mathematical modelling of within-host dynamics of malaria.

\section{References}

[1] R.M. Anderson, R.M. May, and S. Gupta, Non-linear phenomena in host-parasite interactions, Parasitology 99 (1989), pp. S59-S79.

[2] K. Artvanis-tsakonas, J.E. Tongren, and E.M. Riley, The war between the malaria parasite and the immune system: Immunity, immunoregulation and immunopathology, Clin. Exp. Immunol. 133 (2003), pp. 145-152. 
[3] N.T.J. Bailey, The biomathematics of malaria, in Malaria. Charles Griffin \& Co. Ltd, London, 1982, pp. 1-93.

[4] C.A. Bate, J. Tarvene, and J.H.L. Playfair, Malarial parasites induce TNF production by macrophages, Immunology 64 (1988), pp. 227-231.

[5] I.A. Clark, J.L. Virelizier, E.A. Carswell, and P.R. Wood, Possible importance of macrophage-derived mediators in acute malaria, Infect. Immun. 32 (1981), pp. 1058-1066.

[6] P. De Leenheer and H.L. Smith, Virus dynamics: A global analysis, SIAM J. Appl. Math. 63 (2003), pp. 1313-1327.

[7] H.H. Diebner, M. Eincher, L. Molineaux, W.E. Collins, G.M. Jeffert, and K. Dietz, Modelling the transition of asexual blood stages of Plasmodium falciparum to gametocytes, J. Theor. Biol. 202 (2000), pp. 113-127.

[8] O. Diekmann, J.A.P. Heesterbeek, and J.A.J. Metz, On the definition and computation of the basic reproduction ratio $R_{0}$ in models for infectious diseases in heterogeneous population, J. Math. Biol. 28 (1990), pp. $365-382$.

[9] M.M. Elloso, H.C. van der Heyde, J.A. vande Waa, D.D. Manning, and W.P. Weidanz, Inhibition of Plasmodium falciparum in vitro by human gamma delta T cells, J. Immunol. 153 (1994), pp. 1187-1194.

[10] C.R. Engwerda, and M.F. Good, Interactions between malaria parasites and the host immune system, Curr. Opin. Immunol. 17 (2005), pp. 381-387.

[11] M. Fan, M.Y. Li, and K. Wang, Global stability of an SEIS epidemic model with recruitment and a varying total population size, Math. Biosci. 170 (2001), pp. 199-208.

[12] G. Fritsche, C. Larcher, H. Schennach, and G. Weiss, Regulatory interactions between iron and nitric oxide metabolism for immune defense against Plasmodium falciparum infection, J. Infect. Dis. 183 (2001), pp. $1388-1394$.

[13] V.V. Ganusov, C.T. Bergstrom, and R. Antia, Within-host dynamics and the evolution of microparasites in a heterogeneous host population, Evolution 56(2) (2002), pp. 213-223.

[14] M.F. Good, H. Xu, M. Wykes, and C.R. Engwerda, Development and regulation of cell-mediated immune responses to the blood stages of malaria: Implications for vaccine research, Annu. Rev. Immunol. 23 (2005), pp. 69-99.

[15] M.B. Gravenor, and D. Kwiatkowski, An analysis of the temperature effects of fever on the intra-host population dynamics of Plasmodium falciparum, Parasitology 117 (1998), pp. 97-105.

[16] M.B. Gravenor, A.R. McLean, and D. Kwiatkowski, The regulation of malaria parasitaemia: Parameter estimates for a population model, Parasitology 110 (1995), pp. 115-122.

[17] M.B. Gravenor, A.L. Lloyd, P.G. Kremsner, M.A. Missinou, M. English, K. Marsh, and D. Kwiatkowski, A model for estimating total parasite load in Falciparum malaria patients, J. Theor. Biol. 217 (2002), pp. $137-148$.

[18] D. Gurarie and F.E. McKenzie, Dynamics of immune response and drug resistance in malaria infection, Malar. J. 5 (2006), p. 86.

[19] D. Gurarie, P.A. Zimmerman, and C.H. King, Dynamic regulation of single- and mixed-species malaria infection: Insights to specific and non-specific mechanisms of control, J. Theor. Biol. 240 (2006), pp. 185-199.

[20] D.T. Haydon, L. Matthews, R. Timms, and N. Colegrave, Top-down or bottom-up regulation of intra-host blood stage malaria: Do malaria parasites most resemble the dynamics of prey or predator, Proc. R. Soc. Lond. 270 (2003), pp. 289-298.

[21] C. Hertzel and R.M. Anderson, The within-host dynamics of blood stage malaria: Theoretical and experimental studies, Parasitology 113 (1996), pp. 25-38.

[22] M.B. Hoshen, R. Heinrich, W.D. Stein, and H. Ginsburg, Mathematical modelling of the within-host dynamics of Plasmodium falciparum, Parasitology 121 (2000), pp. 227-235.

[23] G.N. Jakeman, A. Saul, W.L. Hogarth, and W.E. Collins, Anaemia of acute malaria infections in nonimmune patients primarily results from destruction of uninfected erythrocytes, Parasitology 119 (1999), pp. $127-133$.

[24] K. Kirk, Membrane transport in the malaria-infected erythrocyte, Physiol. Rev. 81 (2001), pp. 495-537.

[25] R.J. Kosinski, Antigenic variation in trypanosomes: A computer analysis of variant order, Parasitology 80 (1980), pp. 343-357.

[26] D. Kwiatkowski, Malaria toxins and the regulation of parasite density, Parasitol. Today 11 (1995), pp. 206-212.

[27] J.P. LaSalle, The Stability of Dynamical Systems. SIAM, Philadelphia, PA, 1976.

[28] W. Ma, Y. Takeuchi, T. Hara, and E. Beretta, Permanence of an SIR epidemic model with distributed time delays, Tokohu Math. J. 54 (2002), pp. 581-591.

[29] D.P. Mason and F.E. McKenzie, Blood-stage dynamics and clinical implications of mixed Plasmodium vivax-Plasmodium falciparum infections, Am. J. Trop. Med. Hyg. 61 (1999), pp. 367-374.

[30] F.E. McKenzie and W.H. Bossert, The dynamics of Plasmodium falciparum blood stage infection, J. Theor. Biol. 188 (1997), pp. 127-140.

[31] P.G. McQueen and F.E. McKenzie, Age-structured red blood cell susceptibility and the dynamics of malaria infections, Proc. Natl. Acad. Sci. USA 101 (2004), pp. 9161-9166.

[32] L. Molineaux and K. Dietz, Review of intra-host models of malaria, Parassitologia 41 (1999), pp. 221-231. 
[33] L. Molineaux, H.H. Diebner, M. Eincher, W.E. Collins, G.M. Jeffery, and K. Dietz, Plasmodium falciparum parasitaemia described by a new mathematical model, Parasitology 122 (2001), pp. 379-391.

[34] J.S. Muldowney, Compound matrices and ordinary differential equations, Rocky Mount. J. Math. 20 (1990), pp. $857-872$.

[35] A. Murase, T. Sasaki, and T. Kagiwara, Stability analysis of pathogen-immune interaction dynamics, J. Math. Biol. 51 (2005), pp. 247-267.

[36] R.A. O'Donnell, T.F. de Koning-Ward, R.A. Burt, M. Bockarie, J.C. Reeder, A.F. Cowman, and B.S. Crabb, Antibodies against merozoite surface protein (MSP)-1(19) are a major component of the invasion-inhibitory response in individuals immune to malaria, J. Exp. Med. 193 (2001), pp. 1403-1412, (247-263).

[37] P. Perlmann and M. Troye-Blomberg, Malaria and the immune system in humans, Malaria Immunol. 80 (2002), pp. 229-242.

[38] K.P. Piper, R.E. Hayward, M.J. Cox, and K.P. Day, Malaria transmission and naturally acquired immunity to PfEMP-1, Infect. Immun. 67 (1999), pp. 6369-6374.

[39] I.M. Rouzine and F.E. McKenzie, Link between immune response and parasite synchronization in malaria, Proc. Natl. Acad. Sci. USA 100 (2003), pp. 3473-3478.

[40] A. Saul, Models for the in-host dynamics of malaria re-visited: Errors in some basic models lead to large overestimates of growth rate, Parasitology 117 (1998), pp. 405-407.

[41] T. Shiri, W. Garira, and S.D. Musekwa, A two strain HIV-1 mathematical model to assess the effects of chemotherapy on disease parameters, J. Math. Biosci. Eng. 2 (2005), pp. 811-832.

[42] M.M. Stevenson and E.M. Riley, Innate immunity to malaria, Nat. Rev. Immunol. 4 (2004), pp. 169-180.

[43] M. Troye-Blomberg, S. Worku, P. Tangteerawatana, R. Jamshaid, K. Soderstrom, G. Elghazali, L. Moretta, M. Hammarstrom, and L. Mincheva-Nilsson, Human gamma delta T cells that inhibit the in vitro growth of the sexual blood stages of the Plasmodium falciparum parasite express cytolytic and proinflammatory molecules, Scand. J. Immunol. 50 (1999), pp. 642-650.

[44] P. van den Driessche and J. Watmough, Reproduction numbers and subthreshold endemic equilibria for compartmental models of disease transmission, Math. Biosci. 180 (2002), pp. 29-48.

[45] N.J. White, Malaria pathophysiology, in Malaria, Parasite Biology, Pathogenesis, and Protection, I.W. Sherman, ed., ASM Press, Washington, DC, 1998, pp. 371-385.

[46] S.N. Wickramasinghe, and S.H. Abdalla, Blood and bone marrow changes in malaria, Bailliers Best Pract. Res. Clin. Haematol. 13 (2000), pp. 277-299.

\section{Appendix A: Global asymptotic stability of the endemic equilibrium state}

Let the endemic equilibrium state of model system (1) be denoted by $E^{\mathrm{e}}=X^{\mathrm{e}}, Y^{\mathrm{e}}, M^{\mathrm{e}}$.

LEMmA A.1. Suppose that $R_{0}>1$ and $\beta \lambda_{X}-\min \left(\mu_{X}, \mu_{Y}\right) \mu_{Y}<0$, then $E^{\mathrm{e}}$ is a globally asymptotically stable steady state for system (1) with respect to initial conditions not on the boundary.

The key to verifying the global stability of $E^{\mathrm{e}}$ is to rule out the existence of periodic solutions [11]. This is achieved by showing that any periodic solution to (1) is orbitally asymptotically stable. Let $p(t) \equiv\left(p_{1}(t), p_{2}(t), p_{3}(t)\right)^{T}$ denote the periodic solution and suppose that its minimal period is $\omega>0$.

Theorem A.2. A sufficient condition for a periodic orbit $\vartheta=\{p(t): 0 \leq t<\omega\}$ of (1) to be asymptotically stable with asymptotic phase is that the periodic linear system

$$
z^{\prime}=\frac{\partial f^{[2]}}{\partial x}(p(t)) z(t)
$$

is asymptotically stable.

$\partial f^{[2]} / \partial x$ is the second compound matrix of system (1). (For detailed discussions of compound matrices we refer the reader to [34]). We will show that

$$
V\left(z_{1}, z_{2}, z_{3}, p(t)\right)=\sup \left\{\left|z_{1}(t)\right|, \frac{p_{2}(t)}{p_{3}(t)}\left(\left|z_{2}(t)\right|+\left|z_{3}(t)\right|\right)\right\}
$$

is a Lyapunov function for system (1). The right-hand derivative of $V(t), D_{+} V$, exists. Direct calculations lead to the following differential inequalities 


$$
D_{+}\left(\left|z_{1}(t)\right|\right) \leq-\left(\beta p_{3}(t)+\mu_{X}+\mu_{Y}\right)\left|z_{1}(t)\right|+\beta \frac{p_{1}(t) p_{3}(t)}{p_{2}(t)} \cdot \frac{p_{2}(t)}{p_{3}(t)}\left(\left|z_{2}(t)\right|+\left|z_{3}(t)\right|\right)
$$

and

$$
\begin{aligned}
D_{+}\left(\frac{p_{2}(t)}{p_{3}(t)}\left(\left|z_{2}(t)\right|+\left|z_{3}(t)\right|\right)\right)= & \left(\frac{p_{2}^{\prime}(t)}{p_{2}(t)}-\frac{p_{3}^{\prime}(t)}{p_{3}(t)}\right) \cdot \frac{p_{2}(t)}{p_{3}(t)}\left(\left|z_{2}(t)\right|+\left|z_{3}(t)\right|\right)+\frac{p_{2}(t)}{p_{3}(t)} D_{+}\left(\left|z_{2}(t)\right|+\left|z_{3}(t)\right|\right) \\
\leq & \left(\frac{p_{2}(t)}{p_{3}(t)}\left(r \mu_{Y}+\beta p_{3}(t)\right)\right)\left|z_{1}(t)\right|-\frac{p_{2}(t)}{p_{3}(t)}\left(\mu_{X}\left|z_{2}(t)\right|+\mu_{Y}\left|z_{3}(t)\right|\right) \\
& +\left(\frac{p_{2}^{\prime}(t)}{p_{2}(t)}-\frac{p_{3}^{\prime}(t)}{p_{3}(t)}-\mu_{\mathrm{M}}-\beta p_{1}(t)\right) \cdot \frac{p_{2}(t)}{p_{3}(t)}\left(\left|z_{2}(t)\right|+\left|z_{3}(t)\right|\right) \\
\leq & \left(\frac{p_{2}(t)}{p_{3}(t)}\left(r \mu_{Y}+\beta p_{3}(t)\right)\right)\left|z_{1}(t)\right| \\
& +\left(\frac{p_{2}^{\prime}(t)}{p_{2}(t)}-\frac{p_{3}^{\prime}(t)}{p_{3}(t)}-\mu_{\mathrm{M}}-\beta p_{1}(t)-\min \left(\mu_{X}, \mu_{Y}\right)\right) \cdot \frac{p_{2}(t)}{p_{3}(t)}\left(\left|z_{2}(t)\right|+\left|z_{3}(t)\right|\right) .
\end{aligned}
$$

Relations (A2) and (A3) lead to

$$
D_{+} V \leq \sup \left\{g_{1}(t), g_{2}(t)\right\},
$$

where

$$
g_{1}(t)=-\left(\mu_{X}+\beta p_{3}(t)\right)+\frac{p_{2}^{\prime}(t)}{p_{2}(t)}, g_{2}(t)=\beta p_{2}(t)+\frac{p_{2}^{\prime}(t)}{p_{2}(t)}-\min \left(\mu_{X}, \mu_{Y}\right) .
$$

It follows from (A5) that $g_{1}(t) \leq-\mu_{X}+p_{2}^{\prime} / p_{2}(t)$, and that $g_{1}(t) \leq g_{2}(t)$, then we have

$$
D_{+} V(t) \leq g_{2}(t) \text {. }
$$

If

$$
\int_{0}^{\omega} g_{2}(t) \mathrm{d} t<0
$$

then it will follow from (A6) that $V$ is a Lyapunov function for system (1) and this will conclude the proof of the theorem. Since $p(t)$ is a solution of (1), we see that

$$
\int_{0}^{\omega} \mu_{Y} p_{2}(t) \mathrm{d} t=\int_{0}^{\omega} \beta p_{1}(t) p_{3}(t) \mathrm{d} t=\int_{0}^{\omega}\left(\lambda_{X}-\mu_{X} p_{1}(t)\right) \mathrm{d} t \leq \lambda_{X} \omega .
$$

Consequently

$$
\int_{0}^{\omega} g_{2}(t) \mathrm{d} t=\int_{0}^{\omega}\left(\beta p_{2}(t)-\min \left(\mu_{X}, \mu_{Y}\right)\right) \mathrm{d} t \leq\left(\beta \frac{\lambda_{X}}{\mu_{Y}}-\min \left(\mu_{X}, \mu_{Y}\right)\right) \omega .
$$

It follows that (A7) holds under the assumption that $\beta \lambda_{X}-\min \left(\mu_{X}, \mu_{Y}\right) \mu_{Y}<0$ holds. 


\section{Appendix B: Positivity of solutions}

THEOREM B.1. Let the initial data be $X(0)=X_{0}>0, Y(0)=Y_{0}>0, M(0)=M_{0}>0, B(0)=$ $B_{0}>0$ and $A(0)=A_{0}>0$. Then solutions $(X(t), Y(t), M(t), B(t), A(t))$ of (2) are always positive for any $t>0$.

Proof. We will perform the proof following ideas by [28]. It is easy to see that $X(t)>0$ for all $t>0$. If not, we assume that there exists a first time $t_{u}$ such that $X\left(t_{u}\right)=0, X^{\prime}\left(t_{u}\right) \leq 0$ and $X(t)>0$, $Y(t)>0, M(t)>0, B(t)>0, A(t)>0$ for $0<t<t_{u}$. It follows from Equation (2.1) that we have

$$
X^{\prime}\left(t_{u}\right)=\lambda_{X}+\sigma Y\left(t_{u}\right)-\frac{\beta X\left(t_{u}\right) M\left(t_{u}\right)}{1+c_{0} A\left(t_{u}\right)}-\mu_{X} X\left(t_{u}\right)-\omega X\left(t_{u}\right) M\left(t_{u}\right) B\left(t_{u}\right)>0,
$$

which is a contradiction. Similarly we can also prove by contradiction from Equation (2.4) that $\mathrm{d} B(t) / \mathrm{d} t=\lambda_{B}>0 \forall t>0$ when there exists a first time $t_{v}$ such that $B\left(t_{v}\right)=0$.

Therefore $X(t)$ and $B(t)$ are always positive.

Assume that there exists some time $t_{1}>0$ such that $Y\left(t_{1}\right)=0$, other variables are positive and $Y(t)>0$ for $t \in\left[0, t_{1}\right)$. Integrating Equation (2.2) from 0 to $t_{1}$ we have

$$
\begin{aligned}
Y\left(t_{1}\right)= & Y(0) \exp \left\{-\int_{0}^{t_{1}}\left(\mu_{Y}+\kappa_{Y} B(\tau)\right) \mathrm{d} \tau\right\}+\exp \left\{-\int_{0}^{t_{1}}\left(\mu_{Y}+\kappa_{Y} B(\tau)\right) \mathrm{d} \tau\right\} \\
& \times \int_{0}^{t_{1}} \frac{\beta X(\tau) M(\tau)}{1+c_{0} A(\tau)} \exp \left\{\int_{0}^{\tau}\left(\mu_{Y}+\kappa_{Y} B(\theta)\right) \mathrm{d} \theta\right\} \mathrm{d} \tau>0 .
\end{aligned}
$$

which contradicts $Y\left(t_{1}\right)=0$.

Assume there is some time $t_{2}>0$ such that $A\left(t_{2}\right)=0$ and $A(t)>0$. Then integrating Equation (2.5) from 0 to $t_{2}$, we have

$$
A\left(t_{2}\right)=A(0) e^{-\mu_{A} t_{2}}+e^{-\mu_{A} t_{2}} \int_{0}^{t_{2}} \frac{\eta B(\tau) M(\tau)}{k_{1}+M(\tau)} \mathrm{d} \tau>0,
$$

which contradicts $A\left(t_{2}\right)=0$. Similarly for $M(t)$, assume that there is some time $t_{3}>0$ such that $M\left(t_{3}\right)=0$ and $M(t)>0$. Then integrating Equation (2.3) from 0 to $t_{3}$, we see that

$$
\begin{aligned}
M\left(t_{3}\right)= & M(0) \exp \left\{-\int_{0}^{t_{3}}\left(\mu_{\mathrm{M}}+\kappa_{\mathrm{M}} B(\tau)+\frac{\beta X(\tau)}{1+c_{0} A(\tau)}\right) \mathrm{d} \tau\right\} \\
& +\exp \left\{-\int_{0}^{t_{3}}\left(\mu_{\mathrm{M}}+\kappa_{\mathrm{M}} B(\tau)+\frac{\beta X(\tau)}{1+c_{0} A(\tau)}\right) \mathrm{d} \tau\right\} \\
& \times \int_{0}^{t_{3}} \frac{r \mu_{Y} Y(\tau)}{1+c_{0} A(\tau)} \exp \left\{\int_{0}^{\tau}\left(\mu_{\mathrm{M}}+\kappa_{\mathrm{M}} B(\theta)+\frac{\beta X(\theta)}{1+c_{0} A(\theta)}\right) \mathrm{d} \theta\right\} \mathrm{d} \tau>0 .
\end{aligned}
$$

which contradicts $M\left(t_{3}\right)=0$.

This implies that the solution $(X(t), Y(t), M(t), B(t), A(t))$ is always positive for $t \geq 0$.

\section{Appendix C: Local stability of the disease-free equilibrium}

TheOrem C.1. If $R_{0}<1$, then the disease free state $E_{0}$ is a locally asymptotically stable state of system (2); if $R_{0}>1$, then it is unstable. 
Proof. To prove local stability of the disease-free equilibrium we need to show that all the eigenvalues of the Jacobian matrix of the system evaluated at the disease-free equilibrium are negative. We linearize the system of Equations (2) at the disease-free state $E_{0}$, and find the Jacobian matrix. By inspection, three of the eigenvalues $-\mu_{X},-\mu_{B},-\mu_{A}$ are easily deduced and the remaining two eigenvalues are obtained from the remaining $2 \times 2$ submatrix

$$
\left(\begin{array}{cc}
-\mu_{Y}-\kappa_{Y} \frac{\lambda_{B}}{\mu_{B}} & \beta \frac{\lambda_{X}}{\mu_{X}} \\
\frac{r \mu_{Y}}{1+c_{1} \frac{\lambda_{B}}{\mu_{B}}} & -\mu_{\mathrm{M}}-\kappa_{\mathrm{M}} \frac{\lambda_{B}}{\mu_{B}}-\beta \frac{\lambda_{X}}{\mu_{X}}
\end{array}\right)
$$

Expanding the characteristic equation of this submatrix we get the following characteristic polynomial

$$
\begin{aligned}
\lambda^{2}+ & \left(\mu_{\mathrm{M}}+\mu_{Y}+\kappa_{Y} \frac{\lambda_{B}}{\mu_{B}}+\kappa_{\mathrm{M}} \frac{\lambda_{B}}{\mu_{B}}+\beta \frac{\lambda_{X}}{\mu_{X}}\right) \lambda+\left(\mu_{\mathrm{M}}+\kappa_{\mathrm{M}} \frac{\lambda_{B}}{\mu_{B}}+\beta \frac{\lambda_{X}}{\mu_{X}}\right) \times\left(\mu_{Y}+\kappa_{Y} \frac{\lambda_{B}}{\mu_{B}}\right) \\
& -\frac{r \beta \mu_{Y} \lambda_{X}}{\mu_{X}\left(1+c_{1} \frac{\lambda_{B}}{\mu_{B}}\right)}=0 .
\end{aligned}
$$

Using the Routh-Hurwitz stability criterion we determine that the two eigenvalues of the submatrix (C1) which correspond to the roots of the characteristic Equation (C2) are negative if

$$
\left(\mu_{\mathrm{M}}+\kappa_{\mathrm{M}} \frac{\lambda_{B}}{\mu_{B}}+\beta \frac{\lambda_{X}}{\mu_{X}}\right)\left(\mu_{Y}+\kappa_{Y} \frac{\lambda_{B}}{\mu_{B}}\right)-\frac{r \beta \mu_{Y} \lambda_{X}}{\mu_{X}\left(1+c_{1} \frac{\lambda_{B}}{\mu_{B}}\right)}>0
$$

Condition (C3) reduces to $R_{0}<1$. Therefore if $R_{0}<1$, then all the eigenvalues are negative which implies that the disease free state is stable if $R_{0}<1$.

\section{Appendix D Global stability of the disease-free equilibrium}

TheOrem D.1. The disease-free equilibrium $\left(\lambda_{X} / \mu_{X}, 0,0, \lambda_{B} / \mu_{B}, 0\right)$ of system (2) is globally asymptotically stable, whenever $R_{0}<1$.

Proof. Consider the following Lyapunov function:

$$
\mathcal{L}=\left(\mu_{\mathrm{M}}+\beta \frac{\lambda_{X}}{\mu_{X}}+\kappa \frac{\lambda_{B}}{\mu_{B}}\right) Y+\beta \frac{\lambda_{X}}{\mu_{X}} M
$$

We note from system of Equations (2) that the minimum values of $A$ and $B$ are 0 and $\lambda_{B} / \mu_{B}$ respectively and since $0 \leq \sigma<1$, the maximum value of $X=\lambda_{X} / \mu_{X}$. 
Therefore the Lyapunov derivative is given by

$$
\begin{aligned}
\mathcal{L}^{\prime}= & \left(\mu_{\mathrm{M}}+\beta \frac{\lambda_{X}}{\mu_{X}}+\kappa_{\mathrm{M}} \frac{\lambda_{B}}{\mu_{B}}\right) Y^{\prime}+\beta \frac{\lambda_{X}}{\mu_{X}} M^{\prime} \\
= & \left(\mu_{\mathrm{M}}+\beta \frac{\lambda_{X}}{\mu_{X}}+\kappa_{\mathrm{M}} \frac{\lambda_{B}}{\mu_{B}}\right)\left(\frac{\beta X M}{1+c_{0} A}-\mu_{Y} Y-\kappa_{Y} B Y\right) \\
& +\left(\frac{r \mu_{Y} Y}{1+c_{1} B}-\mu_{\mathrm{M}} M-\kappa_{\mathrm{M}} B M-\frac{\beta X M}{1+c_{0} A}\right) \times \beta \frac{\lambda_{X}}{\mu_{X}} \\
= & \left(\frac{r \beta \mu_{Y} \lambda_{X}}{\mu_{X}\left(1+c_{1} B\right)}-\left(\mu_{Y}+\kappa_{Y} B\right)\left(\beta \frac{\lambda_{X}}{\mu_{X}}+\mu_{\mathrm{M}}+\kappa_{\mathrm{M}} \frac{\lambda_{B}}{\mu_{B}}\right)\right) Y \\
& +\beta\left(\frac{X}{1+c_{0} A}\left(\mu_{\mathrm{M}}+\kappa_{\mathrm{M}} \frac{\lambda_{B}}{\mu_{B}}\right)-\frac{\lambda_{X}}{\mu_{X}}\left(\mu_{\mathrm{M}}+\kappa_{\mathrm{M}} B\right)\right) M \\
< & \left(\mu_{Y}+\kappa_{Y} \frac{\lambda_{B}}{\mu_{B}}\right)\left(\beta \frac{\lambda_{X}}{\mu_{X}}+\mu_{\mathrm{M}}+\kappa_{\mathrm{M}} \frac{\lambda_{B}}{\mu_{B}}\right)\left(R_{0}-1\right) Y+\beta\left(\mu_{\mathrm{M}}+\kappa_{\mathrm{M}} \frac{\lambda_{B}}{\mu_{B}}\right)\left(X-\frac{\lambda_{X}}{\mu_{X}}\right) M \\
< & 0 \text { for } R_{0}<1 \text { since } X \leq \frac{\lambda_{X}}{\mu_{X}} .
\end{aligned}
$$

Since all the parameters of the model are non-negative, it follows that $\mathcal{L}<0$ for $R_{0}<1$ with $\mathcal{L}^{\prime}=0$ if $Y=M=0$. If $M=0 \Rightarrow A=0$. Hence $\mathcal{L}$ is a Lyapunov function on $\Phi$. Since $\Phi$ is invariant and attracting, it follows that the largest compact invariant set in $\left((X, Y, M, B, A) \in \Phi: \mathcal{L}^{\prime}=0\right)$ is the singleton $\left\{E_{0}\right\}$. LaSalle's Invariance Principle [27] then implies that $E_{0}$ is globally asymptotically stable in $\Phi$. 


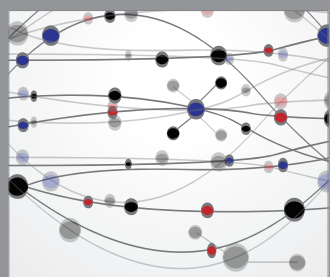

The Scientific World Journal
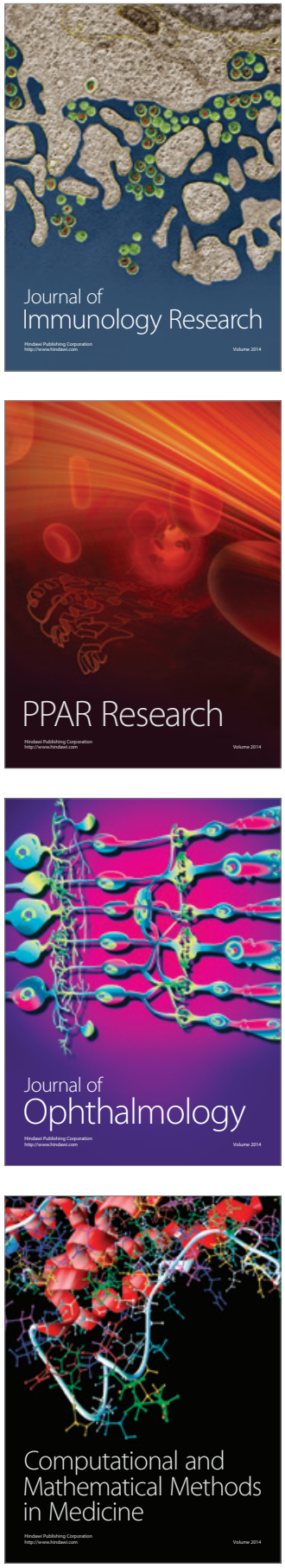

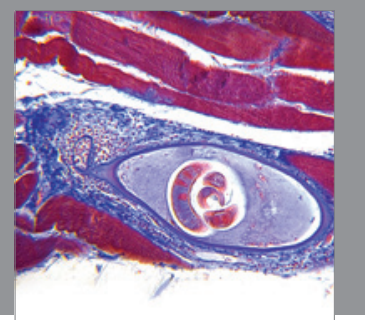

Gastroenterology

Research and Practice
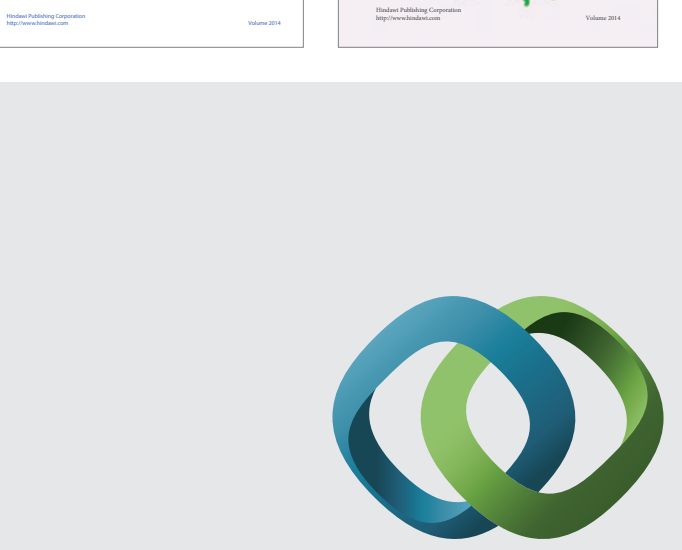

\section{Hindawi}

Submit your manuscripts at

http://www.hindawi.com
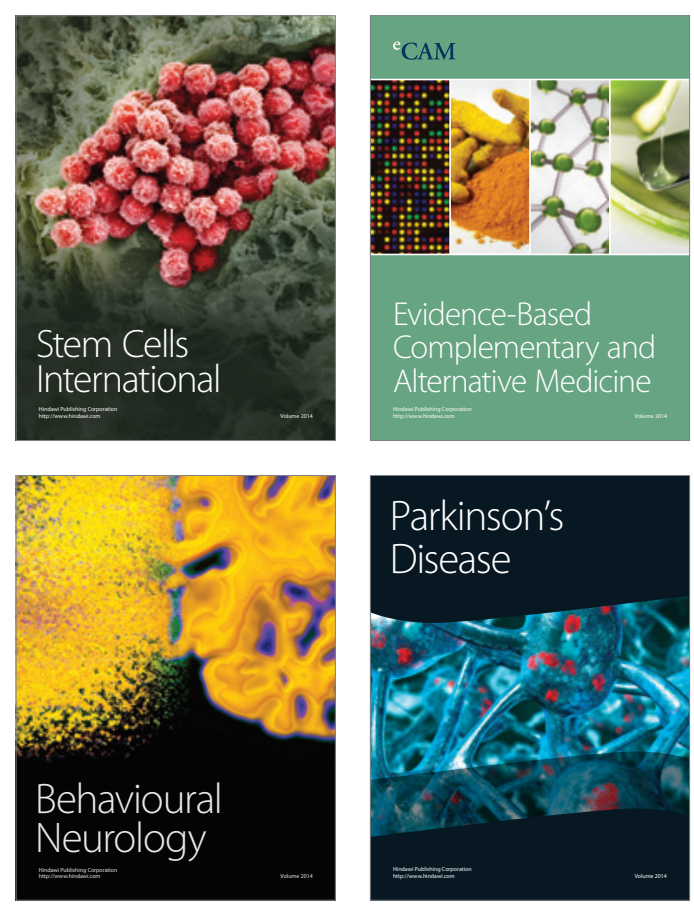

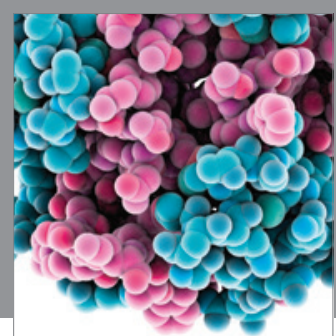

Journal of
Diabetes Research

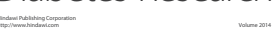

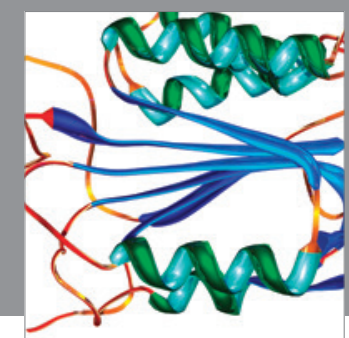

Disease Markers
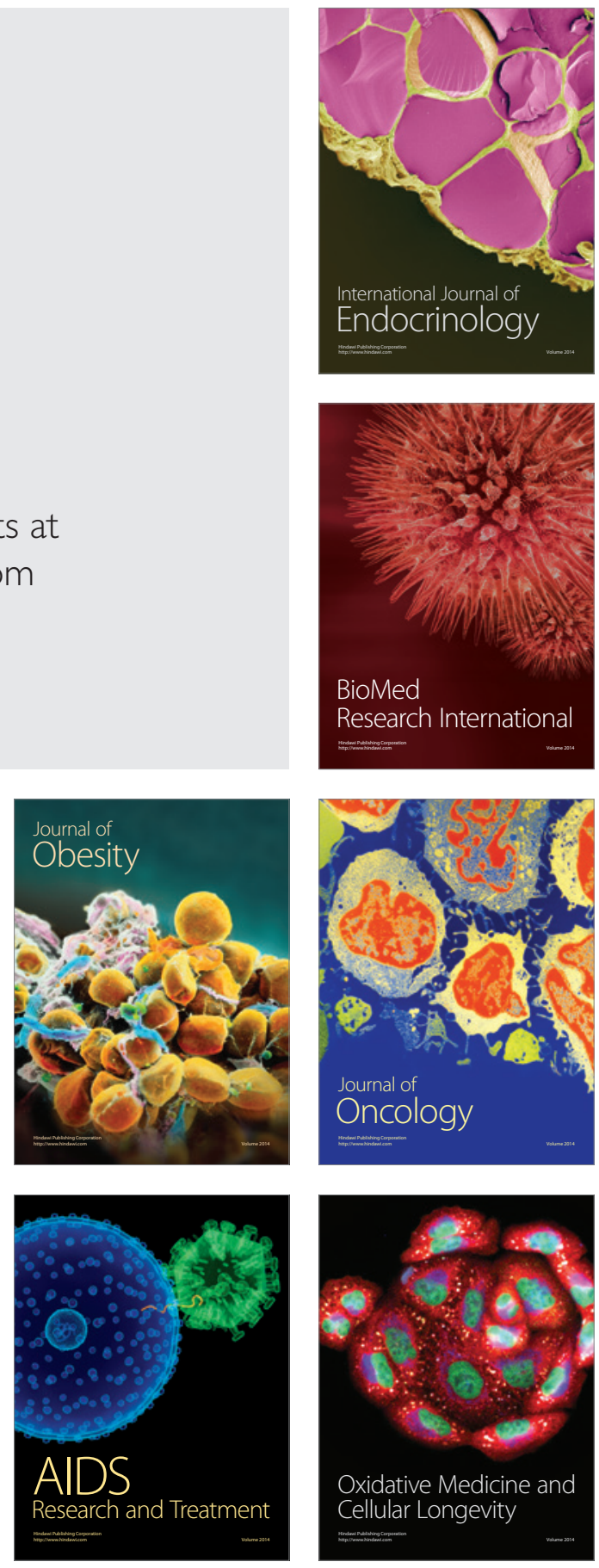